\title{
Assessment of Different Regiments of Early Oral Feeding in Comparison to Delayed Feeding After Elective Uncomplicated Cesarean Section: A Randomized Control Trial
}

\section{Original Article}

\author{
Adel Atef ${ }^{1}$, Rania Elsayed ${ }^{2}$, Akmal El-Mazny ${ }^{1}$ and Sarah Hassan ${ }^{1}$ \\ ${ }^{1}$ Department of Obstetrics and Gynecology, Faculty of Medicine, Cairo University, Egypt \\ ${ }^{2}$ Resident of Obstetrics and Gynecology at Damietta General Hospital, Damietta, Egypt
}

\begin{abstract}
Aim: Starting oral feeding after caesarian section enhances bowel function return, patient's ambulation, and patient recovery. We aimed to evaluate effectiveness of two different approaches of early oral feeding following section on gastrointestinal function, and patient satisfaction.

Materials and Methods: In this randomized control study, 300 pregnant women with elective uncomplicated cesarean section done from October 2019 to March 2020 at Cairo University teaching hospital with were randomly allocated into three feeding groups.

Results: Significant differences were noticed in patients' satisfaction and time interval to return of gastrointestinal function, ambulation, regular diet, and hospital stay between the three groups and between the two groups of early feeding $(P$ value $<0.05$ for all $)$ with highest satisfaction and shortest intervals noticed at introducing soft foods early irrespective of return of intestinal sounds (The first early feeding group). Non-significant differences noted concerning postoperative complications between the three groups or between the two groups of early feeding apart from vomiting found to be higher in the early feeding groups, but the cases were mild and easily treatable.

Conclusion: Early oral feeding reduces the time needed for normal bowel function return and increases satisfaction of the patients with no detrimental significant effects on the gastrointestinal complication.
\end{abstract}

Key Words: Cesarean, early feeding, gastrointestinal symptoms, satisfaction.

Received: 29 July 2021, Accepted: 16 September 2021

Corresponding Author: Adel Atef, 1Department of Obstetrics and Gynecology, Faculty of Medicine, Cairo University, Kasr Alainy Street, Cairo 11562, Egypt, Tel.: +20 1115241250, E-mail: dr.ade190@gmail.com

ISSN: 2090-7265, November 2021, Vol.11, No. 4

\section{INTRODUCTION}

In the industrialized world Caesarean section (CS) is considered one of the major surgical procedures performed commonly in hospitals ${ }^{[1]}$. Rates in Egypt have been steadily increasing to about $52 \%$ in $2014^{[2]}$. With the changing attitudes of surgeons, early oral feeding after abdominal surgery, especially cesarean section, has been considered ${ }^{[3]}$.

Postoperative ileus has become a public health problem because of its role in postoperative morbidity and increased hospital stay ${ }^{[4]}$. Commonly, after abdominal surgery and until return of bowel function (evidenced by bowel sounds, flatus or stool passage, or hunger feeling) no fluids or food are given to patients. This practice is done for prevention of postoperative complications as nausea, vomiting, distention, and others ${ }^{[5]}$. Hence limitation of bowl mobility was believed to follow CS, there was a fear that postoperative ileus will be of common incidence. So that the belief of bowl functions affection after oral feeding was prevalent among both the public and medical staff ${ }^{[6]}$.

From a surgical point, bowel manipulation during cesarean delivery never takes place and peritoneal irritation occurs significantly less often as compared to another laparotomy procedure ${ }^{[7]}$. Interventions as ambulation, probiotics, chewing gum after surgery aimed to accelerate the gastrointestinal motility recovery, and many systematic reviews and clinical trials proved the benefits of early over delayed oral feeding[8]. Early oral feeding may help in reducing rate of body protein depletion, improvement of wound healing and psychological status, and help to reduce the incidence of nosocomial infections, hospital stay time and treatment costs ${ }^{[9]}$.

The current study aimed to evaluate the effect of two different approaches of early oral feeding after cesarean section on gastrointestinal function and patient satisfaction. 


\section{METHODOLOGY}

\section{Study design and setting}

We conducted a randomized controlled study employing a purposeful convenience sample from October 2019 to March 2020. The research was carried out at Labor and Delivery unit at obstetrics and gynaecology department in Kasr Alainy Teaching hospital, located in the metropolitan area of Cairo and serves as the largest tertiary referral center in Egypt and the region of Middle Eas $^{[10]}$. The study has been approved by the research ethics committee, Faculty of Medicine, Cairo University prior to commencement of the study. The current research was prospectively registered in ClinicalTrials.gov registry website with clinical trial identifier (NCT04338737). Informed written consent was obtained from all potential participants following comprehensive explanation of the purpose and potential benefits of the study.

Sample size was calculated using $\mathrm{G}^{*}$ Power software version 3.1.2 for MS Windows, with an error probability of $0.05,80 \%$ for the power and 1 was set for intervention groups ratio resulting in a minimum sample of 100 in each group.

\section{Study population}

All pregnant women, primigravida or had previous one $\mathrm{C} / \mathrm{S}$, aged 20-40 years, with viable singleton pregnancy who planned to undergo elective $\mathrm{c} / \mathrm{s}$ for obstetric indications were eligible to participate in the study. Patients were excluded from the study if they had chronic medical disorders such as diabetes, liver and kidney diseases, history of major abdominal surgery other than $\mathrm{C} / \mathrm{S}$ and intraoperative intestinal surgery. Those who experienced obstructed labor with features suggestive of peritonitis and who declined to participate were also excluded from the study.

Three-hundred women participated in this work were randomly and evenly assigned to early oral feeding (EOF) or late oral feeding (LOF) groups. Patients allocated to EOF were subsequently divided into 2 groups, $\mathrm{A}$ and $\mathrm{B}$. Group A comprised of 100 women received water and clear fluid approximately 2 hours after surgery, followed by soft food and regular diet 4 hours later irrespective to intestinal sounds, flatus, or stool. In the same context, 2 hours post $\mathrm{C} / \mathrm{S}$, water and clear fluid were initiated in group B till the return of intestinal sounds, then soft food, and regular diet later. Group $\mathrm{C}$ participants were made NPO and received 2-3 litres intravenous fluid till the return of bowel functions. Thereafter, water and clear fluids were given followed by soft food and, eventually a regular diet.

\section{Study measurements}

All eligible patients participating in the study were subjected to spinal anaesthesia using heavy Marcaine injected by a spinal needle no 25 . Preoperative prophylactic dose of 3rd generation cephalosporin was administered to all women preceded by skin sensitivity test. All C/S deliveries were performed according to the local hospital protocol by a well-trained obstetrician. We adopted double-layer closure of the uterine incision with gentle manipulation of the tissue ${ }^{[11]}$. Postoperatively, all patients were examined hourly for the first 6 hours then four hourly till the time of patients discharge. First audible intestinal sounds and passage of first flatus, postoperatively, were recorded in hours for all study population. Assessment of possible postoperative complications and/or discomfort such as nausea, vomiting, abdominal distension, and manifestations suggestive of ileus was done. The patient satisfaction was evaluated by all participants using visual analogue scale prior to discharge.

\section{Statistical analysis}

Statistical package for the Social Sciences (SPSS) version 26 (IBM Corp., Armonk, NY, USA) was used for coding and entering data. Summarization of data was done using mean and standard deviation for quantitative variables and frequencies (number of cases) and relative frequencies (percentages) for categorical variables. Analysis of variance (ANOVA) was used for group comparison and post hoc test for multiple comparisons. Chi square $(\chi 2)$ test was used for categorical data comparison. Statistical significance is considered with $P$-values less than 0.05

\section{RESULTS}

In our study 525 women were allocated for the study where 225 women were excluded as 119 women did not fit the inclusion criteria and 86 women met the exclusion criteria and 20 women refused to participate. The 300 women participated in this work were randomly allocated into three equal groups 100 in each.

The mean age in the participant women was $26.88 \pm 3.9$ years, the mean BMI was $29.048 \pm 0.9415 \mathrm{Kg} / \mathrm{M} 2$, the mean parity was $1.84 \pm 0.819$, and the mean operative duration was $44.32 \pm 6.59$ minutes. (Table 1) showed basic demographic characteristics of included participants with no significant difference between the three groups. There was no significant difference between the three groups as regarding presence of adhesions ( $p$ value 0.517 ).

Clear fluid was allowed for Group A and B 2 hours after surgery and only allowed for the third group after open bowel. Semisolid and regular diet were allowed for group A 4 hours after surgery regardless return of intestinal function while for group B semisolid and regular diet were allowed after return of intestinal sound. For group $\mathrm{C}$ semisolid and regular diet were allowed after open bowel after somewhile from clear fluid. So, there was statistically highly significant difference between the three 
groups as regarding post-operative interval to return of bowel sounds ( $p$ value 0.0001 ), return of bowel movement ( $p$ value 0.0001$)$, return of regular diet ( $p$ value 0.0001 ), starting ambulation ( $p$ value 0.0001 ), time to be eligible for discharge ( $p$ value 0.0001), and as regarding satisfaction assessed using the VAS tool at discharge ( $p$ value 0.0001 ) as shown in (Table 2). These results showing that group A had the best outcome as regarding bowel function and satisfaction (Figure 1) and has earlier ambulation and shorter hospital stay (Figure 2) followed by group B and then group $\mathrm{C}$.

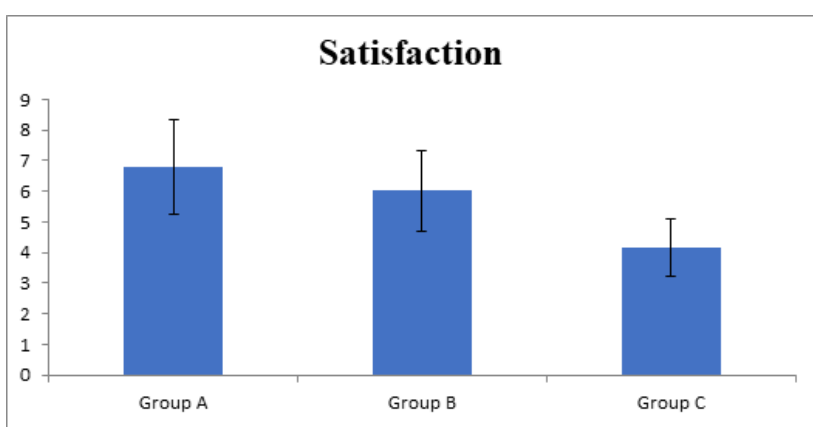

Fig. 1: Participants satisfaction among different groups

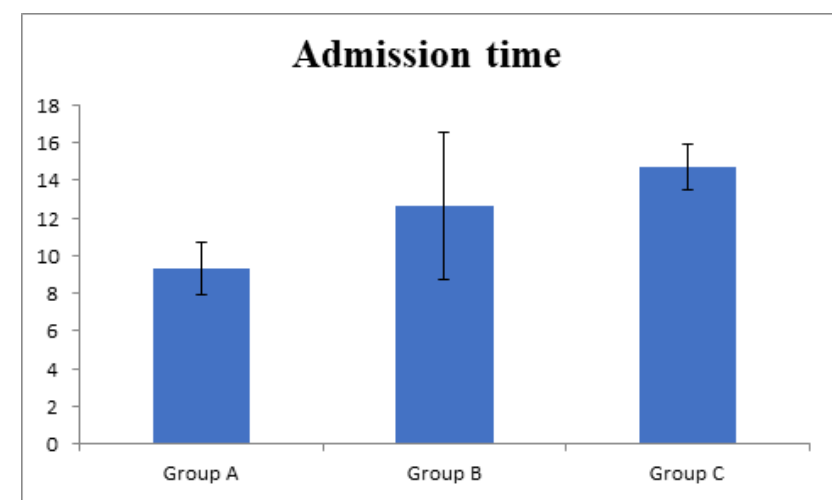

Fig. 2: The hospital stay length among three groups.

Table 1: Demographic data of the studied groups

\begin{tabular}{lccccccc}
\hline & \multicolumn{2}{c}{ Group A } & \multicolumn{2}{c}{ Group B } & \multicolumn{2}{c}{ Group C } \\
\cline { 2 - 7 } & Mean & Standard Deviation & Mean & Standard Deviation & Mean & Standard Deviation & $P$ value \\
\hline Age (years) & 26.47 & 3.72 & 27.42 & 3.33 & 26.75 & 3.37 & 0.141 \\
BMI & 28.96 & 1.06 & 29.18 & 0.89 & 29.01 & 0.86 \\
Parity & 1.82 & 0.86 & 1.95 & 0.82 & 1.75 & 0.77 \\
\hline
\end{tabular}

Table 2: Effect of different approaches on patient's outcome

\begin{tabular}{|c|c|c|c|c|c|c|c|}
\hline & \multicolumn{2}{|r|}{ Group A } & \multicolumn{2}{|c|}{ Group B } & \multicolumn{2}{|r|}{ Group C } & \multirow[b]{2}{*}{$P$ value } \\
\hline & Mean & Standard Deviation & Mean & Standard Deviation & Mean & Standard Deviation & \\
\hline $1^{\text {st }}$ oral fluid feeding (hours) & 2.00 & 0.00 & 2.00 & 0.00 & 10.14 & 0.91 & $<0.001$ \\
\hline Semisolid diet intake (hours) & 4.00 & 0.00 & 5.59 & 0.87 & 10.14 & 0.91 & $<0.001$ \\
\hline Regular diet (hours) & 4.00 & 0.00 & 8.30 & 2.98 & 12.28 & 1.07 & $<0.001$ \\
\hline $1^{\text {st }}$ intestinal sound (hours) & 5.35 & 0.59 & 5.59 & 0.87 & 7.07 & 0.76 & $<0.001$ \\
\hline Open bowel (hours) & 6.08 & 0.94 & 8.30 & 2.98 & 10.14 & 0.91 & $<0.001$ \\
\hline Satisfaction & 6.80 & 1.56 & 6.01 & 1.32 & 4.16 & 0.95 & $<0.001$ \\
\hline Ambulation (hours) & 5.68 & 1.13 & 6.62 & 1.33 & 7.51 & 1.23 & $<0.001$ \\
\hline Admission time (hours) & 9.30 & 1.40 & 12.65 & 3.88 & 14.69 & 1.23 & $<0.001$ \\
\hline
\end{tabular}

significant $P<0.05$ 
Table 3 showed the effect of each feeding approach on unpleasant outcome as nausea where there was no significant difference between the three approaches ( $P$ value 0.27$)$, while significant difference was observed between different groups on vomiting ( $P$ value 0.014$)$, where delayed feeding group has the least number and patients experienced vomiting were received IV metoclopramide and none of them had second attack. As regarding distension, ileus and readmission, there was no statistical difference between the studied groups.

Table 3: Postoperative complications in different groups

\begin{tabular}{|c|c|c|c|c|c|c|c|c|}
\hline & & \multicolumn{2}{|c|}{ Group A } & \multicolumn{2}{|c|}{ Group B } & \multicolumn{2}{|c|}{ Group C } & \multirow[b]{2}{*}{ Pvalue } \\
\hline & & Count & $\%$ & Count & $\%$ & Count & $\%$ & \\
\hline \multirow{2}{*}{ Nausea } & Yes & 16 & $16.0 \%$ & 15 & $15.0 \%$ & 23 & $23.0 \%$ & \multirow{2}{*}{0.276} \\
\hline & No & 84 & $84.0 \%$ & 85 & $85.0 \%$ & 77 & $77.0 \%$ & \\
\hline \multirow{2}{*}{ Vomiting } & Yes & 13 & $13.0 \%$ & 8 & $8.0 \%$ & 2 & $2.0 \%$ & \multirow{2}{*}{0.014} \\
\hline & No & 87 & $87.0 \%$ & 92 & $92.0 \%$ & 98 & $98.0 \%$ & \\
\hline \multirow{2}{*}{ Distension } & Yes & 0 & $0.0 \%$ & 1 & $1.0 \%$ & 0 & $0.0 \%$ & \multirow{2}{*}{1} \\
\hline & No & 100 & $100.0 \%$ & 99 & $99.0 \%$ & 100 & $100.0 \%$ & \\
\hline \multirow{2}{*}{ Ileus } & Yes & 0 & $0.0 \%$ & 1 & $1.0 \%$ & 0 & $0.0 \%$ & \multirow{2}{*}{1} \\
\hline & No & 100 & $100.0 \%$ & 99 & $99.0 \%$ & 100 & $100.0 \%$ & \\
\hline \multirow{2}{*}{ Re admission } & Yes & 0 & $0.0 \%$ & 0 & $0.0 \%$ & 0 & $0.0 \%$ & \multirow{2}{*}{------ } \\
\hline & No & 100 & $100.0 \%$ & 100 & $100.0 \%$ & 100 & $100.0 \%$ & \\
\hline
\end{tabular}

significant $P<0.05$

\section{DISCUSSION}

In the current study, information about the return of bowl function following $\mathrm{C}$-section under spinal anesthesia in both the EOF (groups A\&B) and LOF (group C) was evaluated. Evaluation of this comparison revealed that EOF especially group A is superior to LOF as EOF provoked earlier bowel sound after surgery. Also, no significant difference was found in GI complications between both groups apart from mild vomiting. Finally, the maternal satisfaction increased among EOF.

In this study $2 \mathrm{~h}$ after surgery was chosen for the early feeding groups because of possible complications that may occur during this period such as hemorrhage. Jalilian, et al 2013, and Ahmed et al, 2018, also chose the $2 \mathrm{~h}$ postoperative time for the early feeding group ${ }^{[12,13]}$.

The early feeding groups especially group A had a statistically significant shorter mean time to first bowel sound ( $P$ value 0.0001$)$. This finding was similar with other related studies ${ }^{[9,14-17]}$. The possible variations reported may be related to the difference in both nature of diet in different countries and the time length before initiation 'of oral feeding.

Furthermore, women in the early feeding groups had a statistically significant shorter mean time to first passage of flatus ( $P$ value 0.0001$)$. This was also reported by Teoh 2007 with different mean time to first passage of flatus in each study and this may be due to the different approaches of feeding we used ${ }^{[18]}$. Both Ahmed et al 2018, and Kovavisarach, 2005 found the time until the passage of flatus in the early hydration group was shorter than the delayed hydration group but the difference was statistically insignificant $(P \text { value }>0.05)^{[13,19]}$. Kovavisarach, 2005 was including general and spinal anaesthesia in their study and compared their effect and this may be the cause why the difference is insignificant in his study ${ }^{[19]}$.

In our study, time for return to regular diet was statistically significant shorter among group A $(P$ value $0.0001)$. The same was reported by LR, 2017, and Devi et al, 2015 with different mean time to return to regular diet in each study and this may be due to the different approaches of feeding we used ${ }^{[20,21]}$.

Ahmed et al 2018 found the time until the return to regular diet in the early hydration group was shorter than the delayed hydration group but the difference was statistically insignificant $(P \text { value } 0.296)^{[13]}$. This may be due to difference in the meaning of regular diet between our studies (They considered the regular diet as the 2 nd tolerated solid meal). Our findings regarding return of bowel function may be explained by that gastrointestinal hormonal secretion could be stimulated by food ingestion. Moreover, some reflexes may be stimulated which produces coordination of propulsive activity resulting in beneficial effect on bowel motility ${ }^{[22]}$.

Also, in the early feeding groups especially group A had a statistically significant shorter mean time to ambulation ( $P$ value 0.0001$)$. This was also reported by Mohamed A 2018, and Nantasupha et al. 2016 and this may be because prohibiting oral feeding gave the participant the attitude that they are sick and should be in bed (made them play the role of the patient $)^{[23,24]}$.

In our study, group A had a statistically significant shorter mean time to be eligible for discharge $(P$ value 
$0.0001)$ than other groups. The same was observed by AlGhareeb $2013^{[25]}$. Jalilian et al 2013 found that the time was shorter in the early feeding group than the delayed feeding group, but the difference was insignificant, and they explained this as their hospital protocols did not allow post CS discharge before $48 \mathrm{hrs}$. till achieving other criteria ${ }^{[12]}$.

Statistical comparison between the three groups using the VAS tool at discharge showed that women in the early feeding group especially group A had a significantly higher satisfaction than those in group B \&C ( $P$ value 0.0001$)$. The same was observed from previous studies ${ }^{[17,19]}$. Nantasupha et al. 2016, found that patients' satisfaction scores were similar among the three groups (P value 0.110$)$ ${ }^{[24]}$. This may be due to the small sample size they included (40 participant for each group). Assessment of maternal satisfaction is an essential outcome as it affects postpartum blues, future pregnancies decisions, and reputation of both doctor and hospital.

Most of previous studies together with our study confirmed no difference in GI complications (except for vomiting) associated with EOF groups versus LOF group. Nausea was experienced by $16 \%, 15 \%$ and $23 \%$ among groups $\mathrm{A}, \mathrm{B}$ and $\mathrm{C}$ respectively but with no significant difference either between the three groups $(P$ value $>0.276)$ or between the two groups of early feeding (Pvalue 0.500$)$. These results were parallel to the results of Mawson et al 2019, and Ahmed et al 2018 $8^{[13,15]}$. Teoh 2007 showed more nausea in the early feeding group than the delayed feeding group with no statistical significance ( $P$ value 0.3$)$. This may be explained as Teoh 2007 introduced feeding very early $(0.5 \mathrm{~h} \text { after surgery })^{[18]}$.

Vomiting was observed in $13 \%, 8 \%$ and $2 \%$ of groups $\mathrm{A}, \mathrm{B}$ and $\mathrm{C}$ respectively. With significant difference between the three groups ( $P$ value 0.014$)$ having more vomiting in the early feeding groups but no significant difference between the two groups of early feeding $(P$ value 0.178$)$ but the cases was treated easily and didn't affect maternal satisfaction, prevent progress in the stepping diet, or delay their eligibility from discharge later than the time experienced in other participants. Vomiting in those participants may be due to the effect of pregnancy hormones in delaying the gastric emptying time or may be due to their lower vomiting threshold (personal variation). Unlike that, studies of all, Al-Ghareeb 2013, Mawson et al 2019 and Teoh 2007 showed no significant difference between the early and delayed feeding groups as regarding vomiting with less vomiting in the delayed feeding groups $^{[15,18,25]}$.

In our study, there was no cases of distension, paralytic ileus, or readmission in the three groups. Other studies as LR 2017, and Mohamed A 2018 found that there was no significant difference between the early and delayed feeding groups as regarding distension and ileus $(P$ value $>0.05)$ with less cases in the early feeding groups and the ileus when occurred was found to be mild with no cases of severe ileus ${ }^{[20,23]}$. All the above-mentioned studies had no cases of readmission except one case in one study which was readmitted for a septic wound and not for a cause related to the study.

\section{CONCLUSION}

In conclusion, early feeding within 2 hours after $\mathrm{C}$-section and introduction of regular diet regardless to open bowel was found to be more convenient with best maternal satisfaction and improved bowel function with accepted mild complication and shorter hospital stay and found to be superior to other early feeding modality waiting return of bowel sound for regular diet and by far more favorable than the standard delayed feeding regimen. Further studies including emergency cases and other cesarian section categories should be considered.

\section{AUTHORS' CONTRIBUTIONS}

Dr. AA: supervision of the practical work, writing and final revision of the paper. Prof. AM: final revision of the paper. Dr. SH: assistance in writing and final revision. Dr. RE: responsible for the practical part of the research. All authors read and approved the final manuscript.

\section{CONFLICT OF INTERESTS}

There are no conflicts of Interest.

\section{REFERENCES}

1. Njim T, Tanyitiku BS, Mbanga C. Prevalence, indications and neonatal complications of caesarean deliveries in Cameroon: a systematic review and metaanalysis. Archives of Public Health. 2020 Dec;78:1-0.

2. Shaaban MM, Sayed Ahmed WA, Khadr Z, ElSayed HF. Rising cesarean section rates, a patient's perspective: experience from a high birth rate country. Clin Exp Obstet Gynecol. 2014 Jan 1;41(4):436-9.

3. Mangesi L, Hofmeyr GJ. Early compared with delayed oral fluids and food after caesarean section. Cochrane database of systematic reviews. 2002(3).

4. Chapuis PH, Bokey L, Keshava A, et al . Risk factors for prolonged ileus after resection of colorectal cancer: an observational study of 2400 consecutive patients. Annals of surgery. 2013 May 1;257(5):909-15.

5. Guo J, Long S, Li H, Luo J, Han D, He T. Early versus delayed oral feeding for patients after cesarean. International Journal of Gynecology \& Obstetrics. 2015 Feb 1;128(2):100-5. 
6. Masood SN, Masood Y, Naim U, Masood MF. A randomized comparative trial of early initiation of oral maternal feeding versus conventional oral feeding after cesarean delivery. International Journal of Gynecology \& Obstetrics. 2014 Aug 1;126(2):115-9.

7. Betrán AP, Merialdi M, Lauer JA, et al., Rates of caesarean section: analysis of global, regional and national estimates. Paediatric and perinatal epidemiology. 2007 Mar;21(2):98-113.

8. Wallström A, Frisman GH. Facilitating early recovery of bowel motility after colorectal surgery: a systematic review. Journal of clinical nursing. 2014 Jan;23(1-2):24-44.

9. Gocmen A, Gocmen M, Saraogolu M. Early post-operative feeding after caesarean delivery. J Int Med Res 2002; 30:506-11.

10. El Dib NA. Kasr Al Ainy, the story of a palace that became a medical school. Kasr Al Ainy Medical Journal. 2015 Jan 1;21(1):1.

11. El-Khayat W, Elsharkawi M, Hassan A. A randomized controlled trial of uterine exteriorization versus in situ repair of the uterine incision during cesarean delivery. International Journal of Gynecology \& Obstetrics. 2014 Nov 1;127(2):163-6.

12. Jalilian N, Fakhri M, Keshavarzi F. A randomized clinical trial to compare the post-operative outcomes of early vs. late oral feeding after Cesarean section. Life Science Journal. 2013;10(8s):212-5.

13. Ahmed HA, El-Shahawy AA, Sammour HM. Effect of immediate versus early oral hydration on caesarean section postoperative outcomes: a randomized controlled trial. The Egyptian Journal of Hospital Medicine. 2018 Jul 1;72(8):5072-8.

14. Mba SG, Dim CC, Onah HE, Ezegwui HU, Iyoke CA. Effects of early oral feeding versus delayed feeding on gastrointestinal function of post-caesarean section women in a tertiary hospital in Enugu, Nigeria: A randomized controlled trial. Nigerian journal of clinical practice. 2019 Jul 1;22(7):943.

15. Mawson AL, Bumrungphuet S, Manonai J. A randomized controlled trial comparing early versus late oral feeding after cesarean section under regional anesthesia. International journal of women's health. 2019; 11:519.
16. Hsu YY, Hung HY, Chang SC, Chang YJ. Early oral intake and gastrointestinal function and after caesarean delivery: A systematic review and meta-analysis. Obstet Gynecol 2013; 121:1327-34.

17. Ogbadua AO, Agida TE, Akaba GO, Akitoye OA, Ekele BA. Early versus delayed oral feeding after uncomplicated cesarean section under spinal anesthesia: A randomized controlled trial. Niger J Surg 2018; 24:6-11.

18. Teoh WH, Shah MK, Mah CL. A randomised controlled trial on beneficial effects of early feeding post-Caesarean delivery under regional anaesthesia. Singapore medical journal. 2007 Feb 1;48(2):152.

19. Kovavisarach E, Atthakorn M. Early versus delayed oral feeding after cesarean delivery. International Journal of Gynecology \& Obstetrics. 2005 Jul $1 ; 90(1): 31-4$.

20. LR DL. EARLY VERSUS DELAYED ORAL FEEDING IN PATIENTS AFTER CAESAREAN SECTION. Biomedica. 2017 May 26;31(4):323-7.

21. Devi SS, Pillai SK, Vijayaraghavan J. A comparative study of early versus conventional delay in postoperative oral intake in women undergoing caesarean section under regional anesthesia. Ind J Obs Gyna Res. 2015 Dec 15;2(4):276-82.

22. Zhuang CL, Ye XZ, Zhang CJ, Dong QT, Chen BC, Yu $Z$. Early versus traditional postoperative oral feeding in patients undergoing elective colorectal surgery: a meta-analysis of randomized clinical trials. Digestive surgery. 2013;30(3):225-32.

23. Mohamed AH, kamel Gudia AD. The Effect Of Early Versus Delayed Oral Hydration On Post Cesarean Maternal Outcome And Satisfaction. The Malaysian Journal of Nursing (MJN). 2018 Apr 3;9(4):3-10.

24. Nantasupha C, Ruengkhachorn I, Ruangvutilert P. Effect of conventional diet schedule, early feeding and early feeding plus domperidone on postcesarean diet tolerance: A randomized controlled trial. Journal of Obstetrics and Gynaecology Research. 2016 May;42(5):519-25.

25. Al-Ghareeb SA, Ahmad ER, Turki HA. Effect of early oral hydration on post cesarean outcomes. J Am Sci. 2013;9(8):70-8. 